\title{
Insurance On The Internet: A Different View
}

Michael Theil, (E-mail: theil@wu-wien.ac.at), University of Economics and Business Administration, Austria

\begin{abstract}
We explain the apparent failure to sell insurance over the Internet by drawing on the process of simplification. That is, people tend to use a reduced set of a given decision problem in order to decrease cognitive load. In the context of insurance, this process is shown to result in underestimation of risk, and consequently in low amounts of insurance sold. The solution to this problem lies in making loss information mentally available, for which the multimedia capabilities of the Internet may prove useful.
\end{abstract}

\section{Introduction}

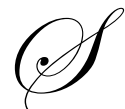

ome years ago, the Internet has been welcomed by insurance enterprises as a new channel of distribution. However, these hopes were not met. Drawing on previous work on the simplification of decision problems, we argue that reduced representations of risk result in underestimation of risk. As a consequence, people take out less than optimal insurance. To counter this effect, mental availability of the menace has to be increased, for instance by vividly describing events of loss.

The paper is organized as follows. In the following section, we will briefly describe the status quo of insurance companies on the Internet. Then, we describe the effects of representativeness and mental availability on risk assessment, concentrating on the consequences for insurance. Finally, we derive the implications for insurance on the Internet.

\section{Status Quo}

By the mid-nineties, insurance enterprises in the United States and in the European Union had to face aggravating market conditions. For companies in the United States increasing global and domestic competition was combined with the persistent pressure to downsize and reduce expenses (Krohm 1996), while in the European Union isolated national markets were largely abandoned in favor of the single European market for insurance.

At about the same time, the Internet became more and more common not only in businesses, but also in households. Therefore, it was just natural to view this new medium of mass communication as a new opportunity for insurance companies. In particular, the World Wide Web, as the most integrative technology on the Internet, was expected to support marketing and production of insurance in various ways (Theil 1996).

Forecasts concerning the penetration of online insurance generally started from growth rates of Internet access, extrapolating these general results to the growth of financial services sold over the Internet, among them insurance. From today's perspective, there were two fundamental problems with these predictions. First, the willingness to conclude financial transactions over the Internet was overestimated. And second, insurance is a quite special kind of financial service that requires particular attention.

Despite ambitious expectations, only about one percent of insurance is sold over the Internet today in the United States (Klauber 2000) and in Western Europe (Holzheu, Trauth and Birkmaier 2000). In fact the existing,

Readers with comments or questions are encouraged to contact the author via email. 
traditional channels of distribution, mostly through insurance agents, still enjoy unbroken popularity. Online sales are mostly limited to smaller policies and few lines of coverage (Prince 1999).

A number of reasons may be responsible for this development. Some argue that customers are simply dissatisfied without specifying the causes more precisely (Maurice 1998). Others complain the lack of saving opportunities, because online policies are not cheaper than others (Spencer 2001). Enormous price differences (Trembly 2001) make insurance offers over the Internet appear dubious.

Some still hope that things will change to the better. Klauber (2000) and Ginarlis (2001) argue that intangible goods would qualify better for online selling than any other products. Therefore, insurance has good prospects of being successful on the Internet. On the other hand, insurance is usually regarded as a very complex and demanding product (Dorfman 1998), which might be a limiting factor.

This point, the complexity of insurance decisions, plays only a marginal role in the current debate concerning online selling of insurance. However, past research has shown that confronted with complex situations, people employ methods of simplification that may lead to a new, sometime completely different perspective.

\section{The Importance of Selling Insurance}

General

A common saying among insurance professionals goes that "Insurance is sold, not bought". In essence, it implies that without active efforts on the side of an insurance company (or state authorities, in some cases), too few (or perhaps the "wrong") policies are taken out. In practice this means that people are often reluctant to purchase insurance for small-probability, high-loss events, which - in theory - would qualify best for being insured.

From the perspective of the insurance enterprise, there is another important aspect of selling: The core business of insuring is to achieve a balanced portfolio of risks. As a consequence, insured risks have to be actively selected. Otherwise, an insurance company could face the problem that only "bad" risks are insured, perhaps an insufficiently low premium.

The central reason why consumers often do not consider insuring lies in risk itself. As we have mentioned above, those risks fit transfer by insurance best that occur comparatively rarely and have a relatively large loss potential. In addition, some kinds of damage are often not apparent, for instance the loss of income in the wake of an accident compared to physical damage or injury. These characteristics of risk together with the features of the risk transfer by insurance are often little understood.

It is well established in decision analysis that facing a complex decision, people tend to reduce cognitive strain by employing strategies of simplification (for example Timmermans 1993). Onken, Hastie and Revelle (1985) explain that decision-makers continue to reduce the number of alternatives until the remaining amount of information requires only little cognitive effort. For Wahlund and Gunnarson (1996), financial decisions are particularly demanding and therefore especially prone to the use of simplified representations.

Simplification has been subject to studies of decision behavior in both, experimental (for instance Mador, Sonsino and Benzion 2000) and naturalistic (for instance Johnson and Bruce 1998) settings. Although simplification is well confirmed as a reality, several questions about the determining cognitive processes are still open (Schweitzer 1999).

\section{Representativeness and Mental Availability}

When judging representativeness of an object, we consider whether this object carries the essential features of objects of a particular class (Tversky and Kahneman 1982a). For example, eagles are less representative of the set of birds than robins, and robins are more representative of birds than of animals in general. 
As a basic rule, judgments by representativeness exhibit what is referred to as regression to the mean (Bazerman 1999). That is, people do not consider the whole range of possible values, for instance a probability distribution, but replace it by some "middle" result, for instance the expected value. This tendency, to regard some average value as most representative, does not only apply to judgments of probability, but also to the assessment of possible outcomes.

In contrast to representativeness, which reflects connotative distance, judgments by mental availability are based on associative distance. Accordingly, instances of large classes and co-occurring events are recalled better and faster and likely occurrences are easier to imagine than unlikely ones (Tversky and Kahneman 1982b).

This way of making estimates is not unreasonable in principle, yet it may be obscured by the effects of media coverage (e.g. O'Guinn and Shrum 1997): For instance, the frequency of seldom but vividly described or recent accidents is often vastly overestimated (Russo and Schoemaker 1990).

The impact of availability can also be described negatively, perhaps as non-availability (Russo and Schoemaker 1992). Slovic, Fischhoff and Lichtenstein (1982), for instance, show that even experts fail to discern that half of the possible reasons for an event in a fault tree are missing. In a closer examination of this problem, Dubé-Rioux and Russo (1988) find that the manipulation of some distant or indirect causes is more difficult to detect than of immediate and direct reasons.

Representativeness and mental availability, as processes of simplification, are expected to be employed alternatively (Tversky and Kahneman 1982b). However, they may lead to quite contrasting results: In short, judgments by representativeness tend to lead to average results, while dramatic or recent events appear to be more mentally available.

There is no general rule, which of these two heuristics dominates the other in a given situation. The results by Chapman and Johnson (1994) suggest that extreme events appear implausible and therefore tend to be ignored or undervalued. Ranyard, Charlton and Williamson (2001) explain that average values carry much lower cognitive load and consequently score higher impact.

What is apparent in any case, is that overall, mental availability has a more narrow time horizon. Recency of an event has been shown to be a decisive factor in judgments by availability. In addition, media attention, even for spectacular events, is most commonly short-lived. Therefore we would conjecture that judgements are made by representativeness in general, unless some exciting information appears, which - for a limited period of time - may overturn this basic result.

\section{Reduced Representations of Insurance}

The fundamental characteristics of insurance decisions described above generally leave much room for biased judgment. For instance, probability judgments and estimation of outcomes have been shown to be prone to considerations by representativeness and, accordingly, exhibit a tendency towards average values. Another example for considerations by representativeness is that after a loss, policyholders tend to cancel insurance contracts (Slovic et al. 1977, Kunreuther et al. 1978) or accept only lower insurance premiums (McClelland, Schulze and Coursey 1993). They interpret this behavior as an indicator that the likelihood of two consecutive losses is judged very low.

Although representativeness is most commonly demonstrated by referring to judgment of likelihood, this heuristic seems to be equally important in forming opinions about loss sizes. Shapira (1995) reports that managers often take average loss sizes as estimators for risk. Kunreuther et al. (1978) obtain similar results for nonprofessional decision-makers.

Similarly, people seem to regard full insurance without limits and deductibles as the most representative extent of risk transfer by insurance (Murray 1972). Full cover is generally preferred even though contracts with limits and deductibles are distinctly cheaper. In an analogy to Ranyard, Charlton and Williamson (2001), we 
conjecture that for specified cover average insurance premiums are considered representative.

Concerning mental availability, it has been shown that people systematically overestimate recent or dramatic events. Substantial research on availability is devoted to flood risks. Kunreuther et al. (1978) find that although people in flood-prone areas are well aware of that peril, they still do not feel concerned unless they or one of their acquaintances have some personal loss experience. Therefore Browne and Hoyt (2000), among others, believe that in addition to event recency or vividness, some personal relationship to the specific peril may stimulate overestimation.

Another aspect of availability is that apparent and immediate kinds of damage are much more likely to be taken into account, compared to either indirect or more remote consequences. Business interruption insurance provides a good example for this aspect. Generally, this kind of insurance is not heavily sold, particularly if compared to corresponding property insurance (Harrison 2001, as an example for many others).

Overall, these examples support the notion that judgments by mental availability generally are highly variable and therefore hardly predictable. The main reason is that the particular situation of the decision-maker may vary considerably.

Obviously, the contrasting effects of representativeness and mental availability generate some tension. In the particular case of assessing the alternatives to assume or to insure a specific risk, this may materialize in a way that risks are normally judged by representativeness and therefore underestimated. This, in turn, would result in comparatively low insurance holdings. If newly available information makes people increasingly concerned, this effect may be reversed, at least for some time. Johnson et al. (1993) provide both, anecdotal and experimental support for this hypothesis. In real-life markets, Zaleskiewicz, Piskorz and Borkowska (2000) also establish evidence that more flood insurance is bought when people have memory of a recent disaster, only to fall back on the initial level later.

\section{Implications for Insurance Enterprises on the Internet}

In the preceding sections, we have assumed that decision-makers, instead of putting all relevant information together, make various arrangements in order to obtain a simplified representation of their decision problem.

Altogether, we expect representativeness to result in a decreased demand for insurance. In detail, this can develop in several ways: Potential loss sizes and loss probabilities tend to be underestimated in presence of the representativeness heuristic. Furthermore, if there are several kinds of damage, people will take immediate consequences into account while paying little or no attention to others. Decision-makers are also likely to show reluctance to take out insurance, if he or she still has to participate in risk bearing either through deductibles, limits or premium upgrades, or if a higher premium than the average is charged right from the beginning.

Contrary to that, judgments by mental availability will probably increase consumers' attention for risk transfer via insurance. In particular, spectacular events have been shown to increase mental availability. Therefore, occurrences involving severe loss or readily perceptible kinds of damage are more easily remembered. The same holds for frequent events. Personal involvement in these events seems to even enhance mental availability. However, this effect is rather short-lived. Generally, when judgments by availability and by representativeness collide, sensibility for extreme events may dominate for some time, but decision-makers will return to their initial overly optimistic - assessment sooner or later.

Generally, if we leave the decision to the customer, routine judgment by representativeness will filter out the rare but severe accidents or events insurance is most suitable for. On the other hand, exceptional circumstances may result in higher mental availability of certain situations. The erratic nature of this matter, however, again leads to a less than optimal decision. If the decision affects a longer period of time, that is, if the consequences will be unveiled not immediately, but long after the decision was made, erroneous judgment is even more probable. 
Insurance markets also mirror this result. Despite its cost, channels of distribution that involve personal communication between salesperson and buyer seem to dominate. From the perspective of simplified problem sets, the advantages of these methods of selling are clear. Given that the client tends to underestimate the risk he or she is facing, the salesperson has the opportunity to immediately respond to the client's doubts. Furthermore, the salesperson may bring illustrative examples or past events to memory, which can be readily interpreted as an increase in mental availability.

The prevalent channels of distribution also give the sales intermediaries the opportunity to take the initiative, which may then promote such insurance products that would not be successful on the market otherwise.

Internet presentations of insurance companies, however, are far from that. As Bölscher, Aschenbrenner and von der Schulenburg (1999) point out, the Internet does not offer personal communication, but there are many features that go beyond the traditional forms of advertising and promoting insurance.

Basically, as we have shown before, a successful strategy must address availability of risk. In the field of insurance, Swiss Re and Munich Re offer a great deal of material concerning various risks, yet as reinsurance companies, this targets at primary insurers in the first place. However, much of the information may suit average customers of insurance companies as well.

Apart from printed material, which is most usually distributed by insurance agents, the multimedia features offered on the Internet present a new perspective. Pictures and short movies may give a new, and perhaps more arousing view of a specific risk. Also background information concerning the causes of loss may enhance mental availability.

The particular shape of online presentation of risk and corresponding insurance certainly depends on the individual characteristics. For instance, the insurance company might inform about the uninsured motorist problem, giving some striking examples such as drivers without or with only tightly limited insurance, hit-and-run drivers or insolvent insurers. However, there may be several different solutions to that problem. In any case, Riepl's law, that an established medium of communication is never completely substituted by a new one, is also expected to hold for the channels of insurance distribution. The challenge is to make best use of the distinctive features of either medium.

\section{Conclusions}

We have shown that simplification of insurance problems brings about underestimation of risk and underinsurance. Traditional channels of distribution offer possibilities to counter this tendency by direct communication with the customer. Online presentation of insurance has other advantages, for instance multimedia capabilities. In order to use the Internet effectively as a marketing instrument, insurers have to exploit this distinctive characteristic, for instance by presenting risk information vividly.

\section{References}

1. $\quad$ Bazerman, Max: (1999) Judgment in Managerial Decision-Making. $4^{\text {th }}$ ed., Wiley, New York et al.

2. Bölscher, Jens, Aschenbrenner, Sebastian and Graf von der Schulenburg, J. -Matthias (1999): Internet as a Medium for Information, Communication and Distribution for the Insurance Industry [translation from German]. Zeitschrift für die gesamte Versicherungswissenschaft 88: 207-214

3. Browne, Mark and Hoyt, Robert: (2000) The Demand for Flood Insurance: Empirical Evidence, Journal of Risk and Uncertainty 20 (3): 291-306

4. Chapman, Gretchen and Johnson, Eric (1994): The Limits of Anchoring; Journal of Behavioral Decision Making 7: 223-242

5. $\quad$ Dorfman, Mark (1998): Risk Management and Insurance. $6^{\text {th }}$ ed. Upper Saddle River, New Jersey. Prentice Hall

6. Dubé-Rioux, Laurette and Russo, Edward (1988): An Availability Bias in Professional Judgment; Journal of Behavioral Decision Making 1: 223-237

7. Ginarlis, John (2001): Financial Services in the Virtual World. Geneva Papers on Risk and Insurance 26 (2): $184-205$

8. Harrison, James (2001): Taking Time Out for Time-element Insurance. American Agent and Broker 73 (3): $44-54$

9. Holzheu, Thomas, Trauth, Thomas, Birkmaier, Ulrike (2000): The Impact of E-business on the Insurance Industry: 
Pressure to adapt - Chance to Reinvent: sigma 5/2000

10. Johnson, Eric, Hershey, John, Meszaros, Jacqueline and Kunreuther, Howard (1993): Framing, Probability Distortions, and Insurance Decisions; Journal of Risk and Uncertainty 7: 35-51

11. Johnson, J. and Bruce, A.: (1998) Risk Strategy Under Task Complexity: A Multivariate Analysis of Behaviour in a Naturalistic Setting; Journal of Behavioral Decision Making 11: 1-17

12. Klauber, Adam (2000): Insurance on the Internet. Risk Management and Insurance Review 3 (1): 45-62

13. Krohm, Gregory (1996): A Survey of Insurance Industry and Regulatory Applications on the Internet. Journal of Insurance Regulation 14 (4): 518-548

14. Kunreuther, Howard, Ginsberg, Ralph, Miller, Louis, Sagi, Philip, Slovic, Paul, Borkan, Bradley and Katz, Norman (1978): Disaster Insurance Protection. Public Policy Lessons; Wiley, New York et al.

15. Mador, Galit, Sonsino, Doron and Benzion, Uri: (2000) On Complexity and Lotteries' Evaluation - Three Experimental Observations; Journal of Economic Psychology 21: 625-637

16. Maurice, Alex (1998): Insurance Frustrates Online Shoppers. National Underwriter Life \& Health / Financial Services Edition December 7, 1998: 40-42

17. McClelland, Gary, Schulze, William and Coursey, Don: (1993) Insurance for Low-Probability Hazards: A Bimodal Response to Unlikely Events; Journal of Risk and Uncertainty 7: 95-116

18. Morgan Stanley Dean Witter (ed.) (1999): The Internet and Financial Services.

19. Murray, Michael: (1972) Empirical Utility Functions and Insurance Consumption Decisions; Journal of Risk and Insurance 39: 31-41

20. Onken, James, Hastie, Reid and Revelle, William: (1985) Individual Differences in the Use of Simplification Strategies in a Complex Decision-making Task; Journal of Experimental Psychology. Human Perception and Performance 11 (1): $14-27$

21. O'Guinn, Thomas and Shrum, L. (1997): The Role of Television in the Construction of Consumer Reality; Journal of Consumer Research 23: 278-294

22. Prince, Michael (1999): Internet Use Grows, But Online Insurance Sales may not. Business Insurance December 6: 14, 26

23. Ranyard, Rob, Charlton, John and Williamson, Janis (2001): The Role of Internal Reference Prices in Consumer's Willingness to Pay Judgments: Thaler's Beer Pricing Task Revisited; Acta Psychologica 106: 265-283

24. Russo, Edward and Schoemaker, Paul (1992): Managing Overconfidence; Sloan Management Review 33 (2): $7-17$

25. Schulte-Noelle, Henning (2001): Technological Changes in IT and Their Influence on Insurance: The Change Ahead (I). Geneva Papers on Risk and Insurance 26 (1): 83-88

26. Schweitzer, Maurice: (1999) The Construction of Mental Accounts in Benefits Decision Making; Benefits Quarterly, $1^{\text {st }}$ quarter 1999: 52-56

27. Shapira, Zur: (1995) Risk Taking. A Managerial Perspective. Russel Sage, New York

28. Slovic, Paul, Fischhoff, Baruch and Lichtenstein, Sarah (1982): Facts Versus Fears. Understanding Perceived Risk; in: Kahneman, Daniel, Slovic, Paul and Tversky, Amos (eds.): Judgment Under Uncertainty. Heuristics and Biases. Cambridge University Press, Cambridge: 463-489

29. Slovic, Paul, Fischhoff, Baruch, Lichtenstein, Sarah, Corrigan, Bernard and Combs, Barbara: (1977) Preference for Insuring Against Probable Small Losses: Insurance Implications; Journal of Risk and Insurance 44: 237-258

30. Spencer, Vikki (2001): Online Sales: The "Bleeding Edge". Canadian Underwriter May 2001: 42-44

31. Theil, Michael (1996): Insurance Companies Providing Information in the World Wide Web - Potential Applications and Present Standard [translation from German]. Journal für Betriebswirtschaft 46 (3): 155-164

32. Timmermans, Danielle: (1993) The Impact of Task Complexity on Information Use in Multi-Attribute Decision Making; Journal of Behavioral Decision Making 6: 95-111

33. Trembly, Ara (2001): Online Auto Insurance: A Few New Wrinkles Can't Disguise The Same Old Problems. National Underwriter Property \& Casualty / Risk \& Benefits Management Edition E-Business Supplement March 26, 2001, S5S8

34. Tversky, Amos and Kahneman, Daniel (1982a): Judgments of and by Representativeness; in: Kahneman, Daniel, Slovic, Paul and Tversky, Amos (Eds.): Judgment Under Uncertainty: Heuristics and Biases. Cambridge University Press, Cambridge: 84-98

35. Tversky, Amos and Kahneman, Daniel (1982b): Availability: A Heuristic for Judging Frequency and Probability; in: Kahneman, Daniel, Slovic, Paul and Tversky, Amos (Eds.): Judgment Under Uncertainty: Heuristics and Biases. Cambridge University Press, Cambridge: 163-178 [abbreviated version of a paper that appeared in Cognitive Psychology, 1973, 4: 207-232]

36. Wahlund, Richard and Gunnarsson, Jonas (1996): Mental Discounting in Financial Strategies. Journal of Economic Psychology 17: 709-730

37. Zaleskiewicz, Tomasz, Piskorz, Zbigniew and Borkowska, Anna (2000) Determinants of Decisions Concerning Insuring Oneself Against the Consequences of Flood; in: Hölzl, Erik (ed.): IAREP / SABE 2000 "Fairness \& Cooperation" Conference Proceedings: 442-446. 